\title{
Tinnitus: network pathophysiology-network pharmacology
}

\author{
Ana B. Elgoyhen ${ }^{1 *}$, Berthold Langguth ${ }^{2}$, Sven Vanneste ${ }^{3}$ and Dirk De Ridder ${ }^{3}$ \\ Instituto de Investigaciones en Ingeniería Genética y Biología Molecular, Consejo Nacional de Investigaciones Científicas y Técnicas and Tercera Cátedra de \\ Farmacología, Facultad de Medicina, Universidad de Buenos Aires, Buenos Aires, Argentina \\ 2 Interdisciplinary Tinnitus Clinic, Departments of Psychiatry and Psychotherapy, University of Regensburg, Regensburg, Germany \\ ${ }^{3}$ TRI, BRAR N and Department of Neurosurgery, University Hospital Antwerp, Edegem, Belgium
}

\section{Edited by:}

Jos J. Eggermont, University

of Calgary, Canada

Reviewed by:

Jos J. Eggermont, University

of Calgary, Canada

Thomas J. Brozoski, Southern Illinois

University School of Medicine, USA

\section{*Correspondence:}

Ana B. Elgoyhen, Instituto de

Investigaciones en Ingeniería

Genética y Biología Molecular,

Consejo Nacionla de Investigaciones

Científicas y Técnicas, Vuelta de

Obligado 2490, 1428 Buenos Aires,

Argentina.

e-mail: elgoyhen@dna.uba.ar,

abelgoyhen@gmail.com
Tinnitus, the phantom perception of sound, is a prevalent disorder. One in 10 adults has clinically significant subjective tinnitus, and for one in 100, tinnitus severely affects their quality of life. Despite the significant unmet clinical need for a safe and effective drug targeting tinnitus relief, there is currently not a single Food and Drug Administration (FDA)-approved drug on the market. The search for drugs that target tinnitus is hampered by the lack of a deep knowledge of the underlying neural substrates of this pathology. Recent studies are increasingly demonstrating that, as described for other central nervous system (CNS) disorders, tinnitus is a pathology of brain networks. The application of graph theoretical analysis to brain networks has recently provided new information concerning their topology, their robustness and their vulnerability to attacks. Moreover, the philosophy behind drug design and pharmacotherapy in CNS pathologies is changing from that of "magic bullets" that target individual chemoreceptors or "disease-causing genes" into that of "magic shotguns," "promiscuous" or "dirty drugs" that target "disease-causing networks," also known as network pharmacology. In the present work we provide some insight into how this knowledge could be applied to tinnitus pathophysiology and pharmacotherapy.

Keywords: graph analysis, brain networks, network pharmacology, phantom percept, tinnitus, small-world, scale-free, magic bullets

\section{TINNITUS PHARMACOTHERAPY: WHERE DO WE STAND?}

Tinnitus, the phantom perception of sound, represents a highly prevalent and distressing condition. Although most cases of tinnitus derive from deprivation of auditory input, it goes beyond the classical definition of an otologic illness, since it encompasses a range of symptoms that are likely to place a huge burden on patients and significantly impair quality of life (Jastreboff, 1990). This can include irritability, agitation, stress, insomnia, anxiety, and depression. In fact, for one in 100 adults, tinnitus affects their ability to lead a normal day-to-day life (Vio and Holme, 2005). Estimates indicate that 13 million people in Western Europe and the USA currently seek medical advice for their tinnitus (Vio and Holme, 2005).

The quest toward finding a drug that targets tinnitus has not been that fulfilling. Although a wide variety of compounds is used off-label to treat tinnitus patients, there is still no US Food and Drug Administration (FDA) or European Medicines Agency (EMA) approved drug on the market. The list of used compounds includes anticonvulsants, anxiolytic, antidepressants, NMDA antagonists, cholinergic antagonists, antihistamines, vasodilators, antipsychotics, and calcium antagonists, to name a few (Langguth et al., 2009; Elgoyhen and Langguth, 2010). In some cases, the rationale behind the use of them is to treat the co-morbidities that come along with tinnitus, like depression and anxiety (Johnson et al., 1993; Sullivan et al., 1993; Bahmad et al., 2006). In others, it is derived from the use of drugs which are effective in disorders thought to share some commonalities with tinnitus, like anticonvulsants used in epilepsy
(Hoekstra et al., 2011) and the calcium antagonist gabapentin used in neuropathic pain (Bauer and Brozoski, 2007). Even further, some drugs are used based on known underlying neuronal changes thought to be a neural correlate of tinnitus. Such is the case of NMDA receptor antagonists (Azevedo and Figueiredo, 2007; Figueiredo et al., 2008; Suckfull et al., 2011) and GABA agonists (Johnson et al., 1993; Gananca et al., 2002; Azevedo and Figueiredo, 2007), used with the hope of reversing the increased neuronal excitability observed in several regions of the auditory pathway (Eggermont and Roberts, 2004). Some drugs have been reported to provide moderate relief of symptoms in a subset of patients. However, most drugs have not proven sufficient effectiveness in randomized controlled clinical trials in order to be approved and marketed specifically for tinnitus (Langguth et al., 2009; Elgoyhen and Langguth, 2010; Langguth and Elgoyhen, 2011).

Thus, novel pharmacological approaches for treating tinnitus are required in order to address a widely recognized, yet largely underserved, and unmet, clinical need. Although early on classified as an auditory problem, recent work is indicating that tinnitus is a central nervous system (CNS) disorder, where dynamic multiple parallel overlapping brain networks are involved (Eggermont and Roberts, 2004; Schlee et al., 2009a,b; De Ridder et al., 2011a). Thus, strategies followed in the development of drugs for other CNS pathologies might give some insight into possible avenues in the design of tinnitus pharmacotherapies. In the present work we review some recent trends in the discovery of CNS acting drugs, describe new ways of analyzing brain networks 
in health and disease and propose how this knowledge could be extrapolated to tinnitus.

\section{DRUG DISCOVERY IN CNS DISORDERS}

Serendipity has played a major role in the initial discovery of CNS acting compounds, like the first psychotropic drugs that led to modern pharmacological treatment of psychiatric diseases (Ban, 2006). Although a detailed understanding of the pathophysiology and etiology of CNS disorders remains elusive, the last decade has witnessed a huge leap in our understanding of the basic biological processes that contribute to many human disorders. However, this has not been paralled by an increase in the number of approved new molecular entities. From 1950 to 2008, the FDA approved 1222 new drugs including biologicals (Munos, 2009). Although the investment of pharmaceutical industries in research and development has grown from US $\$ 2$ to $\$ 50$ billion/year from 1980 to 2005 (Conn and Roth, 2008; Paul et al., 2010), the number of approved drugs per year, about 25 , is not greater than 50 years ago. Moreover, of those 25, only two were for psychiatric diseases in 2009 (Hughes, 2010). Thus, the scale of investment has not been matched by output. In addition, there has not been much innovation (Rask-Andersen et al., 2011). For example, the most widely prescribed antipsychotics olanzapine, risperidone, and quetiapine, share mechanisms of action with clozapine, discovered in the 1950s (Conn and Roth, 2008). Clozapine was developed as a chlorpromazine analog, whose antipsychotic actions were discovered serendipitously when being used as a preanesthetic agent in psychiatric patients (Delay et al., 1952). For more modern antipsychotics (with the exceptions of aripiprazole and the substituted benzamides) a major goal has been to create clozapine-like compounds, devoid of its more serious side effects (Roth et al., 2004; Conn and Roth, 2008). A similar scenario can be seen in the case of drugs used for anxiety, depression, and epilepsy. Thus, the gold standard of drug discovery as from the 1960s has been the design of more selective drugs with ideally one specific target, with the aim of reducing side effects (Roth et al., 2004; Hopkins, 2007, 2008; Conn and Roth, 2008). However, over the past decade, there has been a significant decrease in the rate by which new drug candidates translated into effective clinical therapies. Even more striking, there has been a worrying rise in late-stage phase 2 and phase 3 attrition, that is leading to a reduction in revenues and a financial shock to the pharmaceutical industry (Kola and
Landis, 2004; Hopkins, 2007, 2008). This might derive from the innermost strategy behind modern drug design: drugs selective for a single molecular target, the "one gene, one drug, one disease" paradigm best known as the Paul Ehrlich's "magic bullet" concept of chemotherapy (Kaufmann, 2008), without acknowledging the network structure of the brain and the properties and behavior of real world networks as described in the following sections.

\section{COMPLEX REAL WORLD NETWORKS}

Reductionism dominated biological research during the last century and provided a wealth of information regarding the individual cellular components and their functions. However, biological functions can rarely be attributed to an individual molecule. Instead, they are the consequence of complex interactions between the cell's numerous constituents, such as proteins, DNA, RNA, small molecules, and of intercellular interactions (Kitano, 2002; Barabasi and Oltvai, 2004). Therefore, a key challenge for biology in the twenty-first century is to understand the structure and the dynamics of the complex intra- and intercellular web of interactions that contribute to the structure and function of a living cell, organ or organism (Barabasi and Oltvai, 2004). Thus, reductionism has made its way to holism and systems biology has emerged as a scientific discipline. It is based upon the notion that all the properties, function and/or behavior of a given system cannot be determined or explained by the properties and function of its component parts alone. Instead, the system as a whole determines in an important way how the parts behave and as a result new properties emerge (Kitano, 2002). These "emergent" properties cannot be predicted a priori, based on the properties of the individual elements. Such is the case of the mind and of mind states like consciousness, emergent properties of the brain occurring between multiple physical and functional levels (Gazzaniga, 2010; Bassett and Gazzaniga, 2011).

An important milestone over the past decade has been the understanding that the structure and evolution of networks appearing in social, technological, and natural systems over time follows a number of basic and reproducible organizing principles, which can be explained by the application of graph theoretical analysis to describe network properties (Albert and Barabasi, 2002). A graph is an abstract representation of a network where nodes or vertices are connected by links or edges (Figure 1). For more than 40 years scientists treated complex networks as being
A

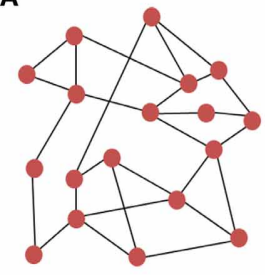

Random Network
B

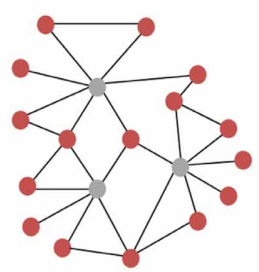

Scale-free Network

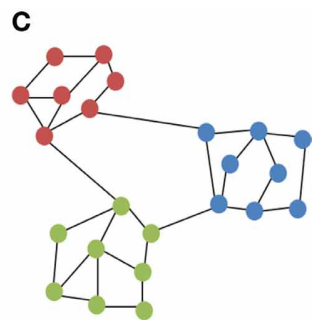

Modular Network
FIGURE 1 | Network topologies. (A) An example of a random network with no high degree hubs, where nodes (red circles) are connected by edges (black lines). (B) A scale-free network with high degree hubs (gray circles).
(C) A modular network where nodes within a module (i.e., red, green, and blue modules) are highly connected to each other and only sparsely connected to nodes of another module. 
random (Erdos and Renyi, 1959). In random graphs, connections between the network nodes are present with a fixed and equal likelihood (Erdos and Renyi, 1959). However, Watts and Strogatz (1998) demonstrated that most real world networks are not random nor regular lattices, but follow the "small-world" phenomenon, where the path length between nodes (the average of the shortest distance between pairs of nodes counted in number of edges) is small, like in random networks, but the clustering coefficient (the likelihood that neighbors of a node will also be connected) is high, unlike, random networks. Watts and Strogatz described the small-world properties of networks found in the nervous system of the nematode Caenorhabditis elegans, a social network of actors and the network of power plants in the United States. A second major discovery in real world network topology was presented by Barabasi and Albert (1999). They proposed a model for the growth of a network where the likelihood that newly added edges connect to a node depends upon the degree (number of edges) of this node, following a preferential attachment behavior. Thus, nodes that have a high degree (hubs) are more likely to get even more edges. This is the network equivalent of "the rich getting richer" (Barabasi and Bonabeau, 2003). Networks generated in this way maintain the short path length of small-world networks (Cohen and Havlin, 2003), but are characterized by a degree distribution described by a power law. These networks are called "scale-free" in the sense that some hubs have a seemingly unlimited number of links and no node is typical of the others (Figure 1). Many other small-world networks have exponential or exponentially truncated power law distributions, implying relatively reduced probabilities of huge hubs (Amaral et al., 2000; Albert and Barabasi, 2002). Determining the topology of a network is important in order to understand the system's behavior, as power laws emerge when there is a transition from disorder to order (Barabasi, 2002). The accidental failure of a number of nodes in a random network can fracture the system into non-communicating islands. In contrast, scale-free networks are resilient to change, have error tolerance and attack vulnerability: they are more robust in the face of random failures or attacks, but they are highly vulnerable to a coordinated attack against their Achilles' heel, the hubs (Albert et al., 2000). These alternate behaviors acquire utmost importance when designing pharmacotherapies for network pathologies.

Since the first description of scale-free networks, most complex networks have been described to have this topology: scientific papers linked by citations, the World Wide Web (Albert et al., 1999; Barabasi and Albert, 1999), e-mail networks (Ebel et al., 2002), epidemic spreads (Pastor-Satorras and Vespignani, 2001), airline transportation networks (Newman, 2003), metabolic, protein-protein interaction and gen interaction networks (Jeong et al., 2000; Podani et al., 2001; Ravasz et al., 2002; Wuchty et al., 2003; Almaas et al., 2004; Barabasi and Oltvai, 2004; Tong et al., 2004), to name a few. But what about brain networks?

\section{BRAIN NETWORKS}

Only recently graph theoretical analysis has been applied to the study of brain networks. These has been motivated by the idea that brain functions are not solely attributable to individual regions and connections, but are emergent features of the topology of the network as a whole, the "connectome" of the brain (Sporns, 2011a). Moreover, it has been boosted by the advancement of the analysis of brain connectivity both at the structural and functional levels (Sporns, 2011a). In this section we will only highlight some important findings and conclusions derived from the application of graph analysis to the topology of brain networks. For comprehensive reviews see Reijneveld et al. (2007), Bullmore and Sporns (2009), Bullmore and Bassett (2011), Sporns (2011a).

Magnetic resonance imaging (MRI) as well as diffusor tensor/spectrum/kurtosis imaging (DTI/DSI/DKI) and the application of graph analysis to some of these data are delivering increasingly detailed maps of large scale human brain structural connectivity and of its topology (Figure 2). In addition, graph analysis to functional connectivity, that is correlated activity in a network, can be applied to data derived from functional MRI (fMRI), magnetoencephalography (MEG), and electroencephalography (EEG). One important caveat to the graph-based study of functional brain organization is how to define the individual nodes that makes up a brain network. If the nodes of the graph do not accurately represent reality then the graph theoretic properties will diverge from the true properties of the system. Therefore, data acquisition and preprocessing are important issues (for review see Bullmore and Bassett, 2011). Effective

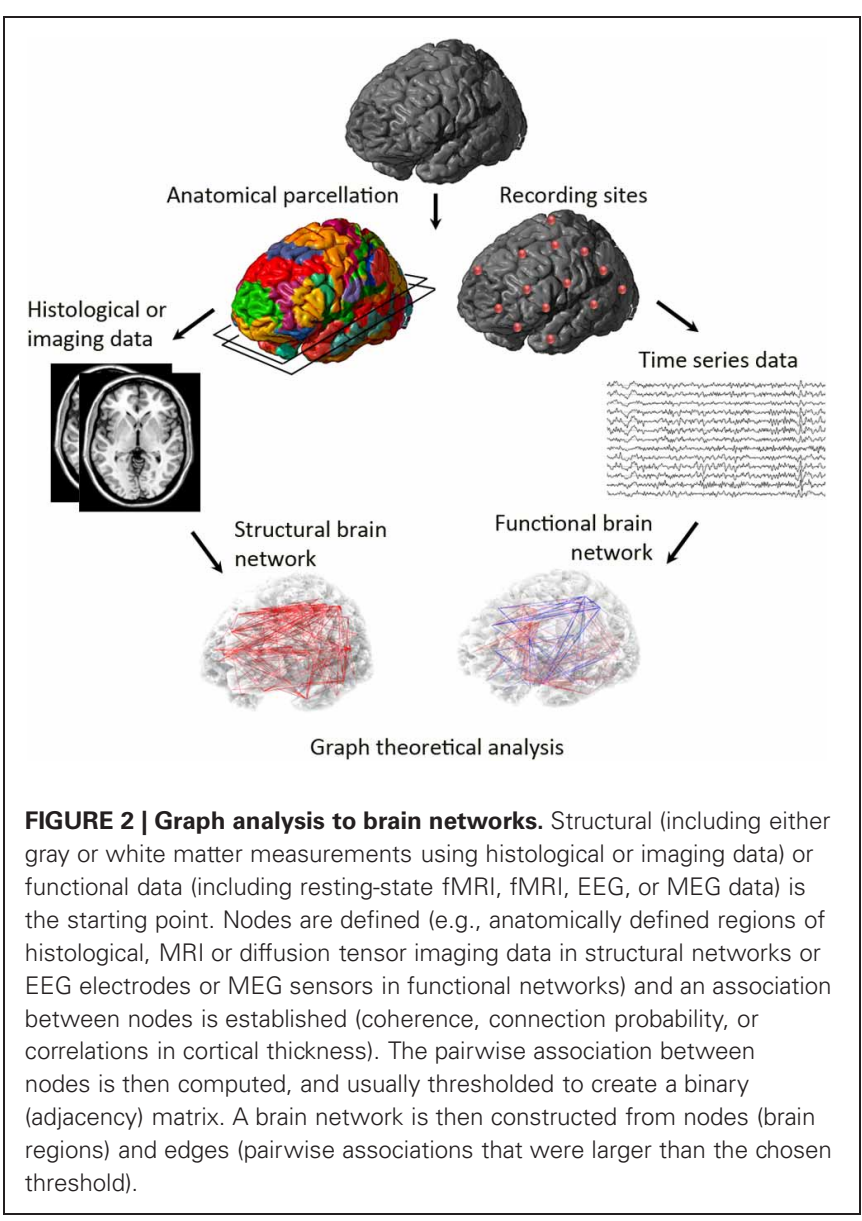


connectivity of brain networks, which refers to information transfer in a network and has some directionality embedded in it, can be analyzed by transfer entropy, Granger causality, or partial directed coherence to generate a directed graph, which involves estimating the causal influence that each element of a system exerts on the behavior of other elements (Sporns, 2011b). However, so far, most graph analyses have been applied to structural and functional connectivity to generate undirected graphs. In addition, although most studies use simpler unweighted graphs, weighted network analysis also has been applied. In these graphs edges can have continuously variable weights indicating the strength or effectiveness of connections (Reijneveld et al., 2007).

Graph analysis of structural and functional connectivity is consistently showing characteristic non-random properties of brain networks (Reijneveld et al., 2007; Bullmore and Sporns, 2009; Bullmore and Bassett, 2011; Sporns, 2011c). At the structural level, several studies have revealed small-world attributes (Bullmore and Sporns, 2009; Sporns, 2011c). Such is the case of the analysis of human brain networks on the basis of correlations in cortical gray matter thickness measured using MRI (He et al., 2007; Chen et al., 2008), DTI, and tractography of cortical and basal brain gray matter areas (Iturria-Medina et al., 2007, 2008; Gong et al., 2009) and diffusion spectrum imaging of cortical regions (Hagmann et al., 2007). In addition, these studies demonstrate the presence of hierarchical brain modules. Modularity refers to the existence of clusters or "network communities" whose constituent brain regions are more densely connected to each other than to regions in other modules (Figure 1). Thus, neurons and brain regions that are spatially close have a relatively high probability of being connected forming a module, whereas connections between spatially remote neurons or brain regions are less likely. Since longer axonal projections are more expensive in terms of their material and energy costs, this layout minimizes wiring costs (Chklovskii et al., 2002). Nodes of high degree, hubs, exist within modules, and also connect to hubs in other modules, thus maintaining the short path length typical of smallworld networks, providing high global efficiency of parallel information transfer (Bullmore and Sporns, 2009; Sporns, 2011c). However, structural brain networks seem to lack extremely high degree nodes characteristic of a scale-free network and rather follow an exponentially truncated power law distribution (He et al., 2007).

Additional information of brain network topology has derived from functional imaging and electrophysiology. Graph analysis from fMRI data has described small-world topology of brain networks, with a truncated power law distribution (Salvador et al., 2005; Achard et al., 2006). In contrast, scale-free topology has been also described in networks derived from fMRI recordings both during task and resting state (Eguiluz et al., 2005; van den Heuvel et al., 2008). Functional connectivity also has been analyzed using a measure of generalized synchronization and then thresholded to generate functional networks, in several studies derived from MEG data sets (Stam and van Dijk, 2002; Stam, 2004). In addition, graph analysis has been applied to wavelet correlation estimates of frequency-dependent functional connectivity between MEG sensors (Bassett et al., 2006). These studies have shown small-world properties of brain networks. Graph analysis to EEG wavelet coefficients found small-world properties in the alpha and beta band networks (Jin et al., 2011). The development of time-varying graphs with fixed nodes but evolving links derived from scalp EEG recordings reported both smallworld and scale-free topology of brain networks (Dimitriadis et al., 2010). Two further studies that have used alternate analysis different from graph theory to EEG recordings, have also shown scale-free properties of brain activity: the analysis of fine temporal structures of arrhythmic brain activity by using nested-frequency EEG analysis (He et al., 2010) and EEG microstates (van de Ville et al., 2010).

From the available structural and functional studies it can be concluded that large-brain networks exhibit a similar organization, with several studies demonstrating functional clusters or modules, highly connected hub nodes, short path lengths, and high global efficiency. The general picture that arises so far from structural studies is that brain networks have small-world topologies but are not scale-free. However, at the functional level it is still a matter of debate whether they are also scale-free (Reijneveld et al., 2007). Differences might derive from the fact that different methods describe different aspects of neuronal networks. From an evolutionary perspective, it can be argued that small-world brain networks have been selected to solve the economic problem of maximizing information processing efficiency, while minimizing wiring costs (Bullmore and Sporns, 2009). Structural studies have revealed important hubs within the parietal and frontal lobes of the cerebral cortex (Iturria-Medina et al., 2008; Gong et al., 2009; van den Heuvel and Sporns, 2011). Most importantly, several independent diffusion imaging data sets have reported a high centrality for the precuneus, the posterior cingulate cortex, and neighboring regions (Hagmann et al., 2007, 2008; Iturria-Medina et al., 2007, 2008). A very recent study that applied graph analysis to DTI data showed the presence of 12 strongly interconnected bihemispheric hub regions, comprising the precuneus, superior frontal, and superior parietal cortex, as well as the subcortical hippocampus, putamen, and thalamus (van den Heuvel and Sporns, 2011). Importantly, these hub regions were not only individually central but formed a "rich club" organization, since they were found to be more densely interconnected than would be expected based only on their degree. This rich club organization of the human brain connectome might optimize global brain communication efficiency for healthy cognitive brain functioning (van den Heuvel and Sporns, 2011).

The analysis of underlying structural and functional network topologies provides a powerful tool to understand the system's behavior that cannot be attained with other approaches to complex systems. Thus, if the brain had a scale-free topology, it would be very vulnerable to hub failures or attacks (Albert et al., 2000). In their analysis of discrete wavelet transform to fMRI time series, Achard and collaborators (2006) described small-world properties of cortical and subcortical regions with a truncated power law distribution, which were as resilient to random error, but more resistant to targeted attacks than scale-free networks. Moreover, up to $40 \%$ of the most connected nodes in the brain network could be eliminated before precipitating a $50 \%$ reduction in size (and twofold increase in path length) of the largest connected 
cluster. Therefore, the small-world architecture of the brain may confer distinctive benefits in terms of robustness to both random elimination of nodes and selective attack on hubs (Achard et al., 2006). Thus, it increases resilience and reduces vulnerability to individual hub attacks. In addition, the rich club organization described by van den Heuvel and Sporns (2011) provides an additional level of resilience to its core, in case of malfunction of any one of its individual key hubs.

\section{BRAIN NETWORKS IN PATHOLOGY}

Only very recently graph theoretical analysis has been applied to a wide variety of CNS disorders and, therefore, the power of this approach toward understanding brain pathology is at its infancy. Changes in network topology have been analyzed in disorders such as schizophrenia, epilepsy, Alzheimer's disease, multiple sclerosis, acute depression, fronto-temporal lobe degeneration, stroke, spinal cord injury, early blindness, and attention deficit hyperactivity disorder (for reviews see Reijneveld et al., 2007; Bassett and Bullmore, 2009; Bullmore and Sporns, 2009).

Reduction in small-world properties has been described in patients with Alzheimer's disease, associated with less efficient information exchange between brain areas, supporting the disconnection hypothesis proposed for this pathology, as well as for many other neurological and psychiatric disorders (Catani and Ffytche, 2005). A loss of small-world properties has also been described in schizophrenia, again supporting the disconnection hypothesis. Network organization derived from graph analysis appears to have increased randomization (Bassett et al., 2008; Lynall et al., 2010; Rubinov and Bassett, 2011) and to be less cost-efficient (Bassett et al., 2009) when compared with healthy controls. In addition, topological abnormalities in people with schizophrenia include a reduced hierarchy of the multimodal cortex (Bassett et al., 2008) and less globally integrated brain structures, with a reduced central role for key frontal hubs, resulting in a limited structural capacity to integrate information across brain regions (Lynall et al., 2010; van den Heuvel et al., 2010). Loss of small-world organization has also been reported in depressed patients during sleep (Leistedt et al., 2009), increased randomization in frontal lobe epilepsy (van Dellen et al., 2009) and shift to more regular networks in children with attention deficit hyperactivity disorder (Wang et al., 2009).

Thus, it seems that in general, brain pathology leads to alteration in small-world network properties with a decreased global integration. Although, it is too soon to fully appreciate the power of graph analysis in CNS disorders, it undoubtedly opens new avenues toward understanding brain diseases. Changes in network topology might be used as clinically useful diagnostic markers and to monitor progression of disease states (Bullmore and Sporns, 2009). Moreover, graph theory allows identifying hubs and modeling network attacks (Albert et al., 2000), which might give hints to determine which treatment approach is the best option at the network level.

\section{NETWORK PHARMACOLOGY}

One major outcome derived from the fact that most complex biological systems are networks, either small-world and/or scale-free, very robust, and resilient to change, has been a very recent shift in the philosophy behind drug design and pharmacotherapy, which is leading to a new trend: in Andrew Hopkins' own words "network pharmacology: the next paradigm in drug discovery" (Hopkins, 2008). This means moving away from Ehrlich's "magic bullets" that target individual chemoreceptors or "disease-causing genes" (Kaufmann, 2008), into "magic shotguns", "promiscuous" or "dirty drugs" that target "diseasecausing networks" (Roth et al., 2004; Csermely et al., 2005; Sams-Dodd, 2005; Hopkins, 2008). Following systems biology principle of emergence, combinations of compounds could be more effective than the sum of the effectiveness of the individual agents themselves (Keith et al., 2005; Kung et al., 2005). Shotguns can make a dramatic impact on disease outcome, as evidenced by the success of the multidrug antiretroviral therapy in decreasing human immunodeficiency virus mortality rates (Imaz et al., 2011). Moreover, multiple attacks have been selected in nature as defense systems and communication, therefore, magic shotguns seem as an evolutionary selected feature. Thus, snake (Bohlen et al., 2011), spider (Rash and Hodgson, 2002; Siemens et al., 2006) cone snail (Olivera and Teichert, 2007) and scorpion (Rodriguez de la Vega et al., 2010) venoms comprise multimolecules, plants employ batteries of various factors to avoid pathogenic attacks (Uma et al., 2011) and honey bee queens produce a mandibular pheromone that is a cocktail of nine interacting components required to attract worker bees, to attract drones for mating and to prevent workers from reproducing (Keeling et al., 2003).

The importance of multi-targeting pharmacology has also recently been encouraged by the observation that novel welltolerated protein kinase drugs, such as Gleevec (Imatinib) and Sutent (SU11248), exhibit binding promiscuity for multiple kinases and therefore are less selective than initially thought (Hampton, 2004; Fabian et al., 2005). Polypharmacology is probably not a novel notion, however, what is new is the acknowledgment of its benefits for efficacy. Thus, the pleiotropic actions of clozapine are probably responsible for its exceptionally beneficial actions in schizophrenia and related disorders. Clozapine has a very complex pharmacological profile, with high affinity for a number of receptors, including dopamine (D4), serotonin (5-HT2A, 5-HT2C, 5-HT6, 5-HT7), muscarinic (M1, M2, M3, M4, M5), adrenergic ( $\alpha 1$ - and $\alpha 2$-subtypes), and other biogenic amine receptors (Roth et al., 2004). Moreover, many newer generation anti-psychotics might have failed in the clinics because of higher target specificity (Roth et al., 2004). Likewise, the pleiotropic actions of antidepressants on signal transduction and neuronal mitogenesis are probably required for the beneficial effects of antidepressants on mood disorders. Moreover, the "dual- and triple-action" antidepressants, which inhibit the reuptake of both 5-HT and other biogenic amines (for example, dopamine and noradrenaline), have been shown to be more effective than "single-action" antidepressants (Roth et al., 2004; Millan, 2006). In a recent study, Yildirim et al. (Yildirim et al., 2007) applied network analysis to 1178 FDA-approved drugs and drugs targets as of March 29, 2006, in order to understand drug design strategies followed by the pharmaceutical industry. To investigate the relationships between approved drugs and 
their targets they built a bipartite drug-target network by integrating publicly available drug data with genetic-disease associations, gene-expression information and protein-protein interaction data. If drugs acted selectively on single targets, one would expect isolated, bipartite nodes and not a network structure. Not surprisingly, the authors found a rich network of polypharmacology interactions between drugs and their targets. Moreover, drugs acting on single targets were the exception. They found a giant component, the largest connected component of the network, with 476 drugs comprising a tightly interconnected neurological drug cluster. Thus, although initially designed as magic bullets, most CNS-acting drugs are magic shotguns and this is probably the reason behind the fact that they are effective in CNS disorders.

The use of multi-targets is further supported by the work of Agoston and collaborators (Agoston et al., 2005). Most studies that have analyzed the stability of networks under failures or attacks have used a model with a complete elimination of an element from the network in order to assess network stability (Albert et al., 2000; Watts, 2002; Shargel et al., 2003; Valente et al., 2004). Agoston and collaborators (Agoston et al., 2005), however, used an alternative approach, where they studied if the partial inactivation of several targets is more efficient than the complete inactivation of a single target. This scenario most closely resembles pharmacotherapy, since at plasma concentrations attained with pharmacological doses, most drugs probably weaken targets rather completely ablate them. Moreover, if only a partial weakening of targets is needed, this could probably be attained at lower plasma concentrations, thus requiring lower doses with concomitant fewer side effects. Partial attacks also mimic other physiological scenarios and treatments, where the complete elimination of a node within a network is a rather unusual phenomenon. By analyzing the regulatory scale-free network of E. coli and S. cerevisiae Agoston and collaborators (2005) concluded that the efficacy of attenuation of targets by multitarget attacks is higher than that of a single-target knockout. In terms of pharmacology, this suggests that drugs with multiple targets or drug combinations might have a better chance to affect the complex equilibrium of the whole system than single target drugs. Moreover, it is sufficient that these multi-target drugs affect their targets only partially, which correlates with the low-affinity interactions of most drugs with several of their targets (Csermely et al., 2005).

Given that promiscuous or dirty drugs are probably more efficient than highly selective ones, can they be designed rationally? In principle, the magic shotgun approach can be attained in four ways: using a drug with multiple mechanisms of actions, prescribing a combination of drugs, the development of multicomponent drugs that contain two or more active ingredients formulated in the same delivery device, or a designer polypharmacology, e.g., a drug with two or more pharmacophores (Borisy et al., 2003; Morphy et al., 2004; Roth et al., 2004; Csermely et al., 2005; Keith et al., 2005; Hopkins, 2007, 2008). The complexity imposed by exploring dosage ranging, drug interaction, and safety studies may significantly raise the practical cost and complexity of developing combination therapies. Potential drug interactions at the pharmacokinetic and pharmacodynamic level have to be considered, since two drugs that themselves are efficient and safe when prescribed separately might not necessarily be efficient and safe when used in combination (Hopkins et al., 2006). However, these problems can be reduced with polypharmacology, since it allows combination therapies at lower doses, resulting in higher efficacy and/or reduced side-effects compared to monotherapies (Morphy et al., 2004; Keith et al., 2005). For example, low-dose combinations of calcium-channel blockers and angiotensin-receptor antagonists are effective for the treatment of hypertension (Andreadis et al., 2005) and low doses of atypical antipsychotics, such as quetiapine, olanzapine, or risperidone, can improve the antidepressant efficacy of selective serotonin reuptake inhibitors, such as fluoxetine, in the treatment of refractory depressed patients (Rasmussen, 2006). Pharmacodynamic and pharmacokinetic relationship, are substantially less complex if polypharmacological action is derived from a single agent and thus approaches to develop multifunctional drugs with more than one pharmacophore are under way (Morphy et al., 2004). An example is ladostigil (TV3326), a novel neuroprotective agent being investigated for the treatment of neurodegenerative disorders like Alzheimer's disease, Lewy body disease, and Parkinson's disease. It combines the acetylcholinesterase and monoamino oxidase (MAO)-A and - $\mathrm{B}$ activities in one molecule and was developed by combining the active (MAO inhibitory and neuroprotective) pharmacophore of the antiparkinsonian MAO-B inhibitor rasagiline with the carbamate cholinesterase inhibitory moiety of the anti-Alzheimer's drug rivastigmine (Weinstock et al., 2006).

Finding the right combination of targets to aim imposes a further complexity when compared to single target therapies. This is the main challenge faced at present in network pharmacology and the field is still "lost in translation" in trying to understand the meaning and the outreach of this new discipline. Following network biology principles, drug discovery approaches might involve the identification of combinations of small molecules that perturb networks in a desired fashion. Drug combinations have been used with compounds already known to be effective in the disease of interest, or where there is a clear rationale for the combination (Millan, 2006). However, such limited combination testing samples only a tiny fraction of the combinatorial pharmacological space and is unlikely to result in the selection of optimal combinations among the very large number of possibilities. A small number of compounds will provide a very large number of combinations and, therefore, efficient screening methods are needed. High-throughput based behavioral screenings which rely on the semi-automated screening of candidate drugs in broad-based behavioral assays in animals, might be used to screen libraries of compounds and find those combinations which are enriched for activity at CNS targets. These approaches that are increasingly offered by specialized companies, have the advantage that they analyze responses to drugs at the level of entire organisms and, therefore, based in their biological function, without the need of having a lead compound, as needed in in vitro assays (Roth et al., 2004; Millan, 2006). In addition, large scale multielectrode brain recordings in animals now offer unique opportunities to assay spatiotemporal patterns of neuronal assemblies in brain networks (Buzsaki, 2004; 
Lehew and Nicolelis, 2008). Graph analysis applied to these multielectrode array approaches might aid toward defining changes in brain topology and hubs in animal models of disease and restoration of the topology during treatment. These methods can provide insights into network-level mechanisms of action of compounds, even when synapse or receptor-level mechanisms are not understood. In addition, it is likely that graph analysis to structural and functional brain networks will further broaden our understanding of treatment effects, will aid toward the design of new therapeutical approaches and help to decipher how therapeutically effective pharmacotherapeutic treatments act on topologically sub-optimal network configurations in patients (Bullmore and Sporns, 2009). Thus, the potential use of fMRI on optimizing drug development (phMRI, pharmacological MRI) is beginning to be appreciated and promises to be part of a sequence of events that could transform drug development for disorders of the CNS (Honey and Bullmore, 2004; Borsook et al., 2006).

Although the rationale behind the use of magic shotguns, promiscuous or dirty drugs is compelling, pharmaceutical industries are being very slow in conquering these approaches. This is probably due to the fact that it is still a challenge to decipher which combinatorial assembly of targets (nodes) to aim, on the one hand, and which combination of dirty drugs is needed in order to best weaken those nodes, on the other. Moreover, the balancing act of optimizing multiple activities, while minimizing unwanted off-target side effects, is a challenge (Hopkins, 2007, 2008). However, network pharmacology will probably become an essential component of drug-development strategies. Indeed, network concepts have already been applied in drug discovery studies in anti-cancer drugs for example (Azmi et al., 2010). Moreover, drug-target networks linking approved or experimental drugs to their protein targets have helped to organize and visualize the considerable knowledge that exists concerning the interplay between diseases, drug targets and drugs (Yildirim et al., 2007; Keiser et al., 2009). The analysis of these networks have indicated that many drugs can be considered palliative, since they do not target the actual disease-associated proteins but proteins in their network neighborhood (Yildirim et al., 2007) and have aided to predict new molecular targets for known drugs (Keiser et al., 2009). Interestingly, Loging and collaborators (Loging et al., 2007) have suggested a high-throughput electronic-biology approach based on in silico data mining of existing databases and integration of this information for drug discovery. These few examples show the power of network approaches. Examples of how these approaches might be applied to tinnitus are developed in the following section.

\section{BACK TO TINNITUS}

An increasing amount of work is supporting the proposal that tinnitus is a CNS pathology where dynamic, multiple, parallel, and overlapping brain networks are at stake (Jastreboff, 1990; Eggermont and Roberts, 2004; Weisz et al., 2007; Schlee et al., 2009a,b; De Ridder et al., 2011a; Leaver et al., 2011). Thus, results obtained in research animals (Eggermont and Roberts, 2004) and humans (Muhlnickel et al., 1998) have shown cortical map plasticity and reorganization of the primary auditory cortex, which correlates with the intensity of the perceived sound (Muhlnickel et al., 1998). Moreover, increased spontaneous activity and increased neural synchrony in cortical neurons have been reported in several regions of the CNS (Norena and Eggermont, 2003; Eggermont, 2007). In addition, MEG studies have shown that tinnitus is related to gamma band activity in the auditory cortex, along with decreased alpha and increased theta or delta activity (Llinas et al., 1999; Weisz et al., 2005, 2007) and EEG studies have further shown that gamma band activity in the auditory cortex reflects the tinnitus intensity (van der Loo et al., 2009). These map changes and cortical synchronized activity are necessary but probably not sufficient for the conscious perception of the phantom sound, which needs functional connectivity to a network of higher order brain "neuronal global workspace” areas (De Ridder et al., 2011a). In accordance, an MEG study has shown a global tinnitus network of longrange cortical connections outside the central auditory system including the right parietal cortex, the right frontal lobe and the anterior cingulate cortex, which project top-down influences on the primary auditory cortex and thus amplify neuronal activity in the sensory cortex (Schlee et al., 2008, 2009a). Moreover, tinnitus has an affective component, since in some patients it is accompanied by stress, depression, and anxiety. Therefore, in addition to the perceptual network, a distress network is activated which comprises the medial temporal lobe (amygdala and hippocampus), parahippocampal areas, insula, and the anterior cingulate cortex (Vanneste et al., 2010; De Ridder et al., 2011b). Salience and mnemonic networks are also activated, evidenced by enhanced activity of the amygdala in positron emission tomography imaging (Mirz et al., 2000), by changes in the hippocampal area and by transient tinnitus diminution after suppression of the amygdalo-hippocampal complex by amytal (De Ridder et al., 2006, 2011b). Thus, there is compelling evidence for a distributed tinnitus brain network, which includes sensory auditory areas together with cortical regions involved in perceptual, emotional, mnemonic, attentional, and salience functions (De Ridder et al., 2011a).

In spite of the above existent electrophysiological and functional imaging data derived from tinnitus patients, there is no published work describing the application of graph theoretical analysis to this data. Graph analysis to the tinnitus network might complement insight derived from the study of more localized small brain areas. Moreover, it might be worth applying graph analysis to structural networks, since structural deficits in tinnitus patients have been described in limbic and auditory pathways by structural imaging approaches (Lee et al., 2007; Landgrebe et al., 2009; Crippa et al., 2010; Husain et al., 2011; Leaver et al., 2011). A clear analogy exists between phantom pain and tinnitus and the available knowledge concerning phantom pain and neuropathic pain has advanced our understanding of the underlying pathophysiological changes in tinnitus (Moller, 2007; De Ridder et al., 2011a). However, there is no published data showing the application of graph analysis to these pathologies either, therefore, the topology of brain networks in phantom perception remains unknown. Graph theory could help to refine the topology of the tinnitus network, with the identification of nodes and high degree hubs, modules and clusters. Moreover, simulation of 
network attacks might aid toward the design of better treatment strategies.

The global tinnitus network of long-range cortical connections outside the central auditory pathway described by Schlee and collaborators $(2008,2009 a)$ by MEG and phase synchronization in the gamma frequencies, speaks toward functionally integrated distributed brain regions. Synchronization of oscillatory responses in the beta- and gamma-band is involved in a variety of cognitive functions, such as routing of signals across distributed cortical networks, perceptual grouping, attention-dependent stimulus selection, sensory-motor integration, working memory and perceptual awareness (Uhlhaas and Singer, 2006). Thus, synchronization plays a crucial role in the exchange of information between cortical areas and both phase and strength of neuronal oscillations in the gamma frequency band influence the amount and speed of information transfer (Buehlmann and Deco, 2010). The global tinnitus network of long-range cortical connections resembles the global neuronal workspace model, where neurons distributed in distant cortical areas need to be accessed for conscious perception (Dehaene and Naccache, 2001; Baars, 2002, 2005). Interestingly, the topology of the global neuronal workspace has been just recently analyzed by graph theoretical analysis during a cognitive effortful task (Kitzbichler et al., 2011). Emergence of a less clustered and less modular network configuration, more globally efficient, with more long-distance synchronization between brain regions, especially in functional networks oscillating at beta and gamma frequency intervals, was reported. This indicates that entrance to the global neuronal workspace breaks modularity in order to allow human brain functional networks to transiently adopt a more efficient but less economical configuration (Kitzbichler et al., 2011). Moreover, the authors propose that, as cognitive effort increases, emergent long-range synchronization provides topological short-cuts between cortical areas that are otherwise segregated from each other in the more modular configuration of the network under cognitively non-demanding conditions and, therefore, increases the global efficiency of the network to subserve transfer of parallel information. This finding is in accordance with the dynamic model of global workspace formation, in which modular subsystems with a locally synchronized community structure during unconscious processing are suddenly replaced by the ignition of a globally synchronized network of neurons with long-range axons densely distributed in prefrontal, parieto-temporal, and cingulate cortices, in response to a consciously attended stimulus (Dehaene and Changeux, 2005, 2011). Thus, following the graph analysis of the neuronal global workspace one could predict a non-random tinnitus network with more long-distance synchronization between brain regions and shorter path lengths which increase global efficiency of information transfer. Interestingly, several independent graph analysis to structural studies in normal subjects have reported a high centrality for the precuneus, the posterior cingulate cortex and neighboring regions (Hagmann et al., 2007, 2008; Iturria-Medina et al., 2007, 2008), two regions that have been shown to be part of the tinnitus network (Schlee et al., 2009b; Vanneste et al., 2010; De Ridder et al., 2011b). Since the precuneus and the posterior cingulate cortex are both pivotal for conscious information processing
(Dehaene and Changeux, 2011), it might be the case that they acquire a higher degree in the phantom percept network.

Being a complex network pathology, tinnitus treatment would best benefit from a promiscuous or multi-target drug approach. This is supported by a recent report in which deanxit, the combination of the antidepressant melitracen and the antipsychotic flupentixol, has proven superior to placebo in a cross-over trial as add-on medication to clonazepam (Meeus et al., 2011). Although there is no tinnitus approved drug on the market, one serendipitous discovery in tinnitus pharmacotherapy deserves further analysis. Beginning with the accidental discovery of the tinnitus suppressing effect of the local anesthetic procaine (Bárány, 1935), intravenous administration of local anesthetics such as lidocaine have been used in the treatment of tinnitus (for review see Trellakis et al., 2007). Available data indicate that lidocaine is able to reduce tinnitus in $60 \%$ of patients in a dose-dependent manner (Haginomori et al., 1995; Otsuka et al., 2003; Baguley et al., 2005). Because of poor biological availability after oral administration, lidocaine is not effective when taken orally. Thus, other anti-arrythmics or local anesthetics have been used without much success. This includes tocainide, flecainide, and mexiletine (Blayney et al., 1985; Hulshof and Vermeij, 1985b; Fortnum and Coles, 1991; Dobie, 1999; Trellakis et al., 2007). Moreover, based on the fact that lidocaine blocks voltage-gated sodium channels, some anticonvulsants that also act on voltage-gated sodium channels like carbamazepine have been used in tinnitus, without much success (Donaldson, 1981; Marks et al., 1981; Hulshof and Vermeij, 1985a). This accidental discovery might be further evaluated and acted upon under the light of network analysis. Two examples of network approaches to this observation are described below.

Brain imaging studies have shown changes in activity in several CNS regions after lidocaine infusion (Mirz et al., 1999; Andersson et al., 2000; Reyes et al., 2002). Graph analysis of functional brain networks might help refine the hubs that are mostly influenced by lidocaine in tinnitus-sensitive patients. This might help understand the mechanism of action of this compound and guide further developments. This type of analysis has been applied to the effect of the dopamine receptor subtype 2 antagonist sulpiride, showing that it impairs network efficiency by an effect most clearly localized to dorsal cingulate and lateral temporal cortical hubs (Achard and Bullmore, 2007). Moreoever, these findings have been correlated with changes in the topology of the aging brain (Achard and Bullmore, 2007).

Lidocaine has pleiotropic effects on several proteins aside from the well-known block of voltage-gated sodium channels (Trellakis et al., 2007). Following an e-biology approach (Loging et al., 2007), one could mine existing databases (including, but not restricted to, medline) to define the pharmacological space of lidocaine targets. Moreover, one could follow the same approach to define the pharmacological space of other local anesthetics, antiarrythmics, and anticonvulsants. A drug-target network linking drugs to their protein targets (Yildirim et al., 2007; Keiser et al., 2009) would help organize and visualize these data, define the target interplay for the different compounds and finally make predictions concerning the add-on effects that lidocaine has when compared to compounds that are non-effective in tinnitus. 
These two examples are just a glimpse of how network analysis might lead us forward in our understanding of tinnitus and of its treatment. Most importantly, it will move us further away from a reductionist way of looking at tinnitus. Tinnitus cannot be seen solely as a pathology of increased excitation or decreased inhibition at different relays of the auditory pathway including the cochlea, cochlear nucleus, inferior colliculus, and sensory auditory cortex. Moreover, the notion of emergence of complex systems might guide us further in tinnitus research and pharmacotherapy. Thus, for example, although benzodiazepines are used in tinnitus patients with the aim of increasing inhibitory gabaergic pathways, recent studies have shown that $G_{A B A}$ receptors can be excitatory in the mature cortex depending on the excitability of the network the neuron is embedded in and on the spatiotemporal relationship to other depolarizing stimulus (Gulledge and Stuart, 2003; Szabadics et al., 2006 and references thereof). These findings imply that different brain regions might qualitative and/or quantitative respond differently to drugs. At least in part, this might be due to drug actions on emergent network properties

\section{REFERENCES}

Achard, S., and Bullmore, E. (2007). Efficiency and cost of economical brain functional networks. PLoS Comput. Biol. 3, e17. doi: 10.1371/ journal.pcbi.0030017

Achard, S., Salvador, R., Whitcher, B., Suckling, J., and Bullmore, E. (2006). A resilient, low-frequency, small-world human brain functional network with highly connected association cortical hubs. $J$. Neurosci. 26, 63-72.

Agoston, V., Csermely, P., and Pongor, S. (2005). Multiple weak hits confuse complex systems: a transcriptional regulatory network as an example. Phys. Rev. E Stat. Nonlin. Soft Matter Phys. 71, 051909.

Albert, R., and Barabasi, A. L. (2002). Statistical mechanics of complex networks. Rev. Mod. Phys. 74, 47-98.

Albert, R., Jeong, H., and Barabasi, A. L. (1999). Internet: diameter of the world-wide web. Nature 401, 130-131.

Albert, R., Jeong, H., and Barabasi, A. L. (2000). Error and attack tolerance of complex networks. Nature 406, 378-382.

Almaas, E., Kovacs, B., Vicsek, T., Oltvai, Z. N., and Barabasi, A. L. (2004). Global organization of metabolic fluxes in the bacterium Escherichia coli. Nature 427, 839-843.

Amaral, L. A., Scala, A., Barthelemy, M., and Stanley, H. E. (2000). Classes of small-world networks. Proc. Natl. Acad. Sci. U.S.A. 97, 11149-11152.

Andersson, G., Lyttkens, L., Hirvela, C., Furmark, T., Tillfors, M., and Fredrikson, M. (2000). Regional tus: a PET case study with lidocaine and auditory stimulation. Acta. Otolaryngol. 120, 967-972.

Andreadis, E. A., Tsourous, G. I., Marakomichelakis, G. E., Katsanou, P. M., Fotia, M. E., Vassilopoulos, C. V., and Diamantopoulos, E. J. (2005). High-dose monotherapy vs low-dose combination therapy of calcium channel blockers and angiotensin receptor blockers in mild to moderate hypertension. $J$. Hum. Hypertens. 19, 491-496.

Azevedo, A. A., and Figueiredo, R. R. (2007). Treatment of tinnitus with acamprosate. Prog. Brain Res. 166, 273-277.

Azmi, A. S., Wang, Z., Philip, P. A., Mohammad, R. M., and Sarkar, F. H. (2010). Proof of concept: network and systems biology approaches aid in the discovery of potent anticancer drug combinations. Mol. Cancer Ther. 9, 3137-3144.

Baars, B. J. (2002). The conscious access hypothesis: origins and recent evidence. Trends Cogn. Sci. 6, 47-52.

Baars, B. J. (2005). Global workspace theory of consciousness: toward a cognitive neuroscience of human experience. Prog. Brain Res. 150, 45-53.

Baguley, D. M., Jones, S., Wilkins, I., Axon, P. R., and Moffat, D. A. (2005). The inhibitory effect of intravenous lidocaine infusion on tinnitus after translabyrinthine removal of vestibular schwannoma: a double-blind, placebo-controlled, crossover study. Otol. Neurotol. 26, 169-176. cerebral blood flow during tinni- conferred to neurons as the result of their membership in the network, rather than being solely due to the intrinsic binding of the drug to a specific receptor in the neuron.

\section{CONCLUSIONS}

In summary, graph analysis to complex networks is aiding toward understanding the behavior of the brain in health and disease. Being tinnitus a CNS disorder where multiple parallel overlapping networks are disturbed, graph analysis might aid to identify the topology of the network including its hubs. Since brain networks are best treated with multi-target drugs that attack the diseasecausing network, tinnitus pharmacological treatments could benefit from dirty or promiscuous drugs. Network analysis to tinnitus pathology and treatment might help to look for the right thing, in the right place and at the right time.

\section{ACKNOWLEDGMENTS}

Ana Belén Elgoyhen, Berthold Langguth, and Dirk De Ridder are funded by the Tinnitus Research Initiative.
Bahmad, F. M. Jr., Venosa, A. R., and Oliveira, C. A. (2006). Benzodiazepines and GABAergics in treating severe disabling tinnitus of predominantly cochlear origin. Int. Tinnitus J. 12, 140-144.

Ban, T. A. (2006). The role of serendipity in drug discovery. Dialogues Clin. Neurosci. 8, 335-344.

Barabasi, A. (ed). (2002). Linked: The New Science of Networks. Cambridge: Perseus Publishing.

Barabasi, A. L., and Albert, R. (1999). Emergence of scaling in random networks. Science 286, 509-512.

Barabasi, A. L., and Bonabeau, E. (2003). Scale-free networks. Sci. Am. 288, 60-69.

Barabasi, A. L., and Oltvai, Z. N. (2004). Network biology: understanding the cell's functional organization. Nat. Rev. Genet. 5, 101-113.

Bárány, R. (1935). Die Beeinfiussung des Ohrensausens durch intravenos injezierte Lokalanasthetika. Acta. Otoralyngol. 23, 201-203.

Bassett, D. S., and Bullmore, E. T. (2009). Human brain networks in health and disease. Curr. Opin. Neurol. 22, 340-347.

Bassett, D. S., Bullmore, E., Verchinski, B. A., Mattay, V. S., Weinberger, D. R., and Meyer-Lindenberg, A. (2008). Hierarchical organization of human cortical networks in health and schizophrenia. J. Neurosci. 28, 9239-9248.

Bassett, D. S., Bullmore, E. T., MeyerLindenberg, A., Apud, J. A., Weinberger, D. R., and Coppola, R. (2009). Cognitive fitness of costefficient brain functional networks.
Proc. Natl. Acad. Sci. U.S.A. 106, 11747-11752.

Bassett, D. S., and Gazzaniga, M. S. (2011). Understanding complexity in the human brain. Trends Cogn. Sci. 15, 200-209.

Bassett, D. S., Meyer-Lindenberg, A., Achard, S., Duke, T., and Bullmore, E. (2006). Adaptive reconfiguration of fractal small-world human brain functional networks. Proc. Natl. Acad. Sci. U.S.A. 103, 19518-19523.

Bauer, C. A., and Brozoski, T. J. (2007). Gabapentin. Prog. Brain Res. 166, 287-301.

Blayney, A. W., Phillips, M. S., Guy, A. M., and Colman, B. H. (1985). A sequential double blind crossover trial of tocainide hydrochloride in tinnitus. Clin. Otolaryngol. Allied Sci. 10, 97-101.

Bohlen, C., Chesler, A., Sharif-Naeini, R., Medzihradszky, K., Zhou, S., King, D., Sánchez, E., Burlingame, A., Basbaum, A., and Julius, D. (2011). A heteromeric Texas coral snake toxin targets acid-sensing ion channels to produce pain. Nature 479, 410-414.

Borisy, A. A., Elliott, P. J., Hurst, N. W., Lee, M. S., Lehar, J., Price, E. R., Serbedzija, G., Zimmermann, G. R., Foley, M. A., Stockwell, B. R., and Keith, C. T. (2003). Systematic discovery of multicomponent therapeutics. Proc. Natl. Acad. Sci. U.S.A. 100, 7977-7982.

Borsook, D., Becerra, L., and Hargreaves, R. (2006). A role for fMRI in optimizing CNS drug development. Nat. Rev. Drug Discov. 5, 411-424.

Buehlmann, A., and Deco, G. (2010). Optimal information transfer in 
the cortex through synchronization. PLoS Comput. Biol. 6. doi: 10.1371/journal.pcbi.1000934

Bullmore, E. T., and Bassett, D. S. (2011). Brain graphs: graphical models of the human brain connectome. Annu. Rev. Clin. Psychol. 7, 113-140.

Bullmore, E., and Sporns, O. (2009). Complex brain networks: graph theoretical analysis of structural and functional systems. Nat. Rev. Neurosci. 10, 186-198.

Buzsaki, G. (2004). Large-scale recording of neuronal ensembles. Nat. Neurosci. 7, 446-451.

Catani, M., and Ffytche, D. H. (2005). The rises and falls of disconnection syndromes. Brain 128, 2224-2239.

Chen, Z. J., He, Y., Rosa-Neto, P., Germann, J., and Evans, A. C. (2008). Revealing modular architecture of human brain structural networks by using cortical thickness from MRI. Cereb. Cortex 18, 2374-2381.

Chklovskii, D. B., Schikorski, T., and Stevens, C. F. (2002). Wiring optimization in cortical circuits. Neuron 34, 341-347.

Cohen, R., and Havlin, S. (2003). Scalefree networks are ultrasmall. Phys. Rev. Lett. 90, 058701.

Conn, P. J., and Roth, B. L. (2008). Opportunities and challenges of psychiatric drug discovery: roles for scientists in academic, industry, and government settings. Neuropsychopharmacology 33, 2048-2060.

Crippa, A., Lanting, C. P., Van Dijk, P., and Roerdink, J. B. (2010). A diffusion tensor imaging study on the auditory system and tinnitus. Open Neuroimag. J. 4, 16-25.

Csermely, P., Agoston, V., and Pongor, S. (2005). The efficiency of multitarget drugs: the network approach might help drug design. Trends Pharmacol. Sci. 26, 178-182.

De Ridder, D., Elgoyhen, A. B., Romo, R., and Langguth, B. (2011a). Phantom percepts: tinnitus and pain as persisting aversive memory networks. Proc. Natl. Acad. Sci. U.S.A. 108, 8075-8080.

De Ridder, D., Fransen, H., Francois, O., Sunaert, S., Kovacs, S., and Van De Heyning, P. (2006). Amygdalohippocampal involvement in tinnitus and auditory memory. Acta. Otolaryngol. Suppl. 126, 50-53.

De Ridder, D., Vanneste, S., and Congedo, M. (2011b). The distressed brain: a group blind source separation analysis on tinnitus. PLoS One 6, e24273. doi: 10.1371/journal.pone.0024273
Dehaene, S., and Changeux, J. P. (2005). Ongoing spontaneous activity controls access to consciousness: a neuronal model for inattentional blindness. PLoS Biol. 3, e141. doi: 10.1371/journal.pbio.0030141

Dehaene, S., and Changeux, J. P. (2011). Experimental and theoretical approaches to conscious processing. Neuron 70, 200-227.

Dehaene, S., and Naccache, L. (2001). Towards a cognitive neuroscience of consciousness: basic evidence and a workspace framework. Cognition 79, 1-37.

Delay, J., Deniker, P., and Harl, J. M. (1952). Therapeutic method derived from hiberno-therapy in excitation and agitation states. Ann. Med. Psychol. (Paris) 110, 267-273.

Dimitriadis, S. I., Laskaris, N. A., Tsirka, V., Vourkas, M., Micheloyannis, S., and Fotopoulos, S. (2010). Tracking brain dynamics via time-dependent network analysis. J. Neurosci. Methods 193, 145-155.

Dobie, R. A. (1999). A review of randomized clinical trials in tinnitus. Laryngoscope 109, 1202-1211.

Donaldson, I. (1981). Tegretol: a double blind trial in tinnitus. J. Laryngol. Otol. 95, 947-951.

Ebel, H., Mielsch, L. I., and Bornholdt, S. (2002). Scale-free topology of e-mail networks. Phys. Rev. E Stat. Nonlin. Soft Matter Phys. 66, 035103.

Eggermont, J. J. (2007). Correlated neural activity as the driving force for functional changes in auditory cortex. Hear. Res. 229, 69-80.

Eggermont, J. J., and Roberts, L. E. (2004). The neuroscience of tinnitus. Trends Neurosci. 27, 676-682.

Eguiluz, V. M., Chialvo, D. R., Cecchi, G. A., Baliki, M., and Apkarian, A. V. (2005). Scale-free brain functional networks. Phys. Rev. Lett. 94, 018102.

Elgoyhen, A. B., and Langguth, B. (2010). Pharmacological approaches to the treatment of tinnitus. Drug Discov. Today 15, 300-305.

Erdos, P., and Renyi, A. (1959). On random graphs: I. Publ. Math. 6, 290-297.

Fabian, M. A., Biggs, W. H. 3rd, Treiber, D. K., Atteridge, C. E., Azimioara, M. D., Benedetti, M. G., Carter, T. A., Ciceri, P., Edeen, P. T., Floyd, M., Ford, J. M., Galvin, M., Gerlach, J. L., Grotzfeld, R. M., Herrgard, S., Insko, D. E., Insko, M. A., Lai, A. G., Lelias, J. M., Mehta, S. A., Milanov, Z. V., Velasco, A. M., Wodicka, L. M., Patel, H. K., Zarrinkar, P. P., and Lockhart, D. J. (2005). A small molecule-kinase interaction map for clinical kinase inhibitors. Nat. Biotechnol. 23, 329-336.

Figueiredo, R. R., Langguth, B., Mello De Oliveira, P., and Aparecida De Azevedo, A. (2008). Tinnitus treatment with memantine. Otolaryngol. Head Neck Surg. 138, 492-496.

Fortnum, H. M., and Coles, R. R. (1991). Trial of flecainide acetate in the management of tinnitus. Clin. Otolaryngol. Allied Sci. 16, 93-96.

Gananca, M. M., Caovilla, H. H. Gananca, F. F., Gananca, C. F., Munhoz, M. S., Da Silva, M. L., and Serafini, F. (2002). Clonazepam in the pharmacological treatment of vertigo and tinnitus. Int. Tinnitus $J$. 8, 50-53.

Gazzaniga, M. S. (2010). Neuroscience and the correct level of explanation for understanding mind. An extraterrestrial roams through some neuroscience laboratories and concludes earthlings are not grasping how best to understand the mindbrain interface. Trends Cogn. Sci. 14, 291-292.

Gong, G., He, Y., Concha, L., Lebel, C., Gross, D. W., Evans, A. C., and Beaulieu, C. (2009). Mapping anatomical connectivity patterns of human cerebral cortex using in vivo diffusion tensor imaging tractography. Cereb. Cortex 19, 524-536.

Gulledge, A. T., and Stuart, G. J. (2003). Excitatory actions of GABA in the cortex. Neuron 37, 299-309.

Haginomori, S., Makimoto, K., Araki, M., Kawakami, M., and Takahashi, H. (1995). Effect of lidocaine injection of EOAE in patients with tinnitus. Acta. Otolaryngol. 115, 488-492.

Hagmann, P., Cammoun, L., Gigandet, X., Meuli, R., Honey, C. J., Wedeen, V. J., and Sporns, O. (2008). Mapping the structural core of human cerebral cortex. PLoS Biol. 6, e159. doi: 10.1371/journal.pbio. 0060159

Hagmann, P., Kurant, M., Gigandet, X., Thiran, P., Wedeen, V. J., Meuli, R., and Thiran, J. P. (2007). Mapping human whole-brain structural networks with diffusion MRI. PLoS One 2, e597. doi: 10.1371/journal. pone.0000597

Hampton, T. (2004). "Promiscuous" anticancer drugs that hit multiple targets may thwart resistance. JAMA 292, 419-422.

He, B. J., Zempel, J. M., Snyder, A. Z., and Raichle, M. E. (2010). The temporal structures and functional significance of scale-free brain activity. Neuron 66, 353-369.
He, Y., Chen, Z. J., and Evans, A. C. (2007). Small-world anatomical networks in the human brain revealed by cortical thickness from MRI. Cereb. Cortex 17, 2407-2419.

Hoekstra, C. E., Rynja, S. P., Van Zanten, G. A., and Rovers, M. M. (2011). Anticonvulsants for tinnitus. Cochrane Database Syst. Rev. CD007960.

Honey, G., and Bullmore, E. (2004). Human pharmacological MRI. Trends Pharmacol. Sci. 25, 366-374.

Hopkins, A. L. (2007). Network pharmacology. Nat. Biotechnol. 25, 1110-1111.

Hopkins, A. L. (2008). Network pharmacology: the next paradigm in drug discovery. Nat. Chem. Biol. 4, 682-690.

Hopkins, A. L., Mason, J. S., and Overington, J. P. (2006). Can we rationally design promiscuous drugs? Curr. Opin. Struct. Biol. 16, 127-136.

Hughes, B. (2010). 2009 FDA drug approvals. Nat. Rev. Drug Discov. 9, 89-92.

Hulshof, J. H., and Vermeij, P. (1985a). The value of carbamazepine in the treatment of tinnitus. ORL J. Otorhinolaryngol. Relat. Spec. 47, 262-266.

Hulshof, J. H., and Vermeij, P. (1985b). The value of tocainide in the treatment of tinnitus. A doubleblind controlled study. Arch. Otorhinolaryngol. 241, 279-283.

Husain, F. T., Medina, R. E., Davis, C. W., Szymko-Bennett, Y., Simonyan, K., Pajor, N. M., and Horwitz, B. (2011). Neuroanatomical changes due to hearing loss and chronic tinnitus: a combined VBM and DTI study. Brain Res. 1369, 74-88.

Imaz, A., Falco, V., and Ribera, E. (2011). Antiretroviral salvage therapy for multiclass drug-resistant HIV-1-infected patients: from clinical trials to daily clinical practice. AIDS Rev. 13, 180-193.

Iturria-Medina, Y., Canales-Rodriguez, E. J., Melie-Garcia, L., ValdesHernandez, P. A., Martinez-Montes, E., Aleman-Gomez, Y., and Sanchez-Bornot, J. M. (2007) Characterizing brain anatomical connections using diffusion weighted MRI and graph theory. Neuroimage 36, 645-660.

Iturria-Medina, Y., Sotero, R. C., Canales-Rodriguez, E. J., AlemanGomez, Y., and Melie-Garcia, L. (2008). Studying the human brain anatomical network via diffusionweighted MRI and Graph Theory. Neuroimage 40, 1064-1076. 
Jastreboff, P. J. (1990). Phantom auditory perception (tinnitus): mechanisms of generation and perception. Neurosci. Res. 8, 221-254.

Jeong, H., Tombor, B., Albert, R., Oltvai, Z. N., and Barabasi, A. L. (2000). The large-scale organization of metabolic networks. Nature 407, 651-654.

Jin, S. H., Lin, P., and Hallett, M. (2011). Reorganization of brain functional small-world networks during finger movements. Hum. Brain Mapp.

Johnson, R. M., Brummett, R., and Schleuning, A. (1993). Use of alprazolam for relief of tinnitus. A double-blind study. Arch. Otolaryngol. Head Neck Surg. 119, 842-845.

Kaufmann, S. H. (2008). Paul Ehrlich: founder of chemotherapy. Nat. Rev. Drug Discov. 7, 373.

Keeling, C. I., Slessor, K. N., Higo, H. A., and Winston, M. L. (2003). New components of the honey bee (Apis mellifera L.) queen retinue pheromone. Proc. Natl. Acad. Sci. U.S.A. 100, 4486-4491.

Keiser, M. J., Setola, V., Irwin, J. J., Laggner, C., Abbas, A. I., Hufeisen, S. J., Jensen, N. H., Kuijer, M. B., Matos, R. C., Tran, T. B., Whaley, R., Glennon, R. A., Hert, J., Thomas, K. L., Edwards, D. D., Shoichet, B. K., and Roth, B. L. (2009). Predicting new molecular targets for known drugs. Nature 462, 175-181.

Keith, C. T., Borisy, A. A., and Stockwell, B. R. (2005). Multicomponent therapeutics for networked systems. Nat. Rev. Drug Discov. 4, 71-78.

Kitano, H. (2002). Systems biology: a brief overview. Science 295, 1662-1664.

Kitzbichler, M. G., Henson, R. N., Smith, M. L., Nathan, P. J., and Bullmore, E. T. (2011). Cognitive effort drives workspace configuration of human brain functional networks. J. Neurosci. 31, 8259-8270.

Kola, I., and Landis, J. (2004). Can the pharmaceutical industry reduce attrition rates? Nat. Rev. Drug Discov. 3, 711-715.

Kung, C., Kenski, D. M., Dickerson, S. H., Howson, R. W., Kuyper, L. F., Madhani, H. D., and Shokat, K. M. (2005). Chemical genomic profiling to identify intracellular targets of a multiplex kinase inhibitor. Proc. Natl. Acad. Sci. U.S.A. 102, 3587-3592.

Landgrebe, M., Langguth, B., Rosengarth, K., Braun, S., Koch, A., Kleinjung, T., May, A., De Ridder, D., and Hajak, G. (2009). Structural brain changes in tinnitus: grey matter decrease in auditory and non-auditory brain areas. Neuroimage 46, 213-218.

Langguth, B., and Elgoyhen, A. (2011). Emerging pharmacotherapy of tinnitus. Expert Opin. Emerg. Drugs 16, 603-606.

Langguth, B., Salvi, R., and Elgoyhen, A. B. (2009). Emerging pharmacotherapy of tinnitus. Expert Opin. Emerg. Drugs 14, 687-702.

Leaver, A. M., Renier, L., Chevillet, M. A., Morgan, S., Kim, H. J., and Rauschecker, J. P. (2011). Dysregulation of limbic and auditory networks in tinnitus. Neuron 69, 33-43.

Lee, Y. J., Bae, S. J., Lee, S. H., Lee, J. J., Lee, K. Y., Kim, M. N., Kim, Y. S., Baik, S. K., Woo, S., and Chang, Y. (2007). Evaluation of white matter structures in patients with tinnitus using diffusion tensor imaging. $J$. Clin. Neurosci. 14, 515-519.

Lehew, G., and Nicolelis, M. A. L. (2008). "State-of-the-art microwire array design for chronic neural recordings in behaving animals," in Methods for Neural Ensemble Recordings, 2nd Edn, ed M. A. L. Nicolelis (Boca Raton, FL: CRC Press) Chapter 1.

Leistedt, S. J., Coumans, N., Dumont, M., Lanquart, J. P., Stam, C. J., and Linkowski, P. (2009). Altered sleep brain functional connectivity in acutely depressed patients. Hum. Brain Mapp. 30, 2207-2219.

Llinas, R. R., Ribary, U., Jeanmonod, D., Kronberg, E., and Mitra, P. P. (1999). Thalamocortical dysrhythmia: a neurological and neuropsychiatric syndrome characterized by magnetoencephalography. Proc. Natl. Acad. Sci. U.S.A. 96, 15222-15227.

Loging, W., Harland, L., and WilliamsJones, B. (2007). High-throughput electronic biology: mining information for drug discovery. Nat. Rev. Drug Discov. 6, 220-230.

Lynall, M. E., Bassett, D. S., Kerwin, R., Mckenna, P. J., Kitzbichler, M., Muller, U., and Bullmore, E. (2010). Functional connectivity and brain networks in schizophrenia. J. Neurosci. 30, 9477-9487.

Marks, N. J., Onisiphorou, C., and Trounce, J. R. (1981). The effect of single doses of amylobarbitone sodium and carbamazepine in tinnitus. J. Laryngol. Otol. 95, 941-945.

Meeus, O., De Ridder, D., and Van De Heyning, P. (2011). Administration of the combination clonazepamDeanxit as treatment for tinnitus. Otol. Neurotol. 32, 701-709.

Millan, M. J. (2006). Multi-target strategies for the improved treatment of depressive states: conceptual foundations and neuronal substrates, drug discovery and therapeutic application. Pharmacol. Ther. 110, 135-370.

Mirz, F., Gjedde, A., Ishizu, K., and Pedersen, C. B. (2000). Cortical networks subserving the perception of tinnitus-a PET study. Acta. Otolaryngol. Suppl. 543, 241-243.

Mirz, F., Pedersen, B., Ishizu, K. Johannsen, P., Ovesen, T., StodkildeJorgensen, H., and Gjedde, A. (1999). Positron emission tomography of cortical centers of tinnitus. Hear. Res. 134, 133-144.

Moller, A. R. (2007). Tinnitus and pain. Prog. Brain Res. 166, 47-53.

Morphy, R., Kay, C., and Rankovic, Z. (2004). From magic bullets to designed multiple ligands. Drug Discov. Today 9, 641-651.

Muhlnickel, W., Elbert, T., Taub, E., and Flor, H. (1998). Reorganization of auditory cortex in tinnitus. Proc. Natl. Acad. Sci. U.S.A. 95 10340-10343.

Munos, B. (2009). Lessons from 60 years of pharmaceutical innovation. Nat. Rev. Drug Discov. 8, 959-968.

Newman, M. (2003). The structure and function of complex networks. SIAM Rev. 45, 167-256.

Norena, A. J., and Eggermont, J. J. (2003). Changes in spontaneous neural activity immediately after an acoustic trauma: implications for neural correlates of tinnitus. Hear. Res. 183, 137-153.

Olivera, B. M., and Teichert, R. W. (2007). Diversity of the neurotoxic Conus peptides: a model for concerted pharmacological discovery. Mol. Interv. 7, 251-260.

Otsuka, K., Pulec, J. L., and Suzuki, M. (2003). Assessment of intravenous lidocaine for the treatment of subjective tinnitus. Ear Nose Throat $J$. 82, 781-784.

Pastor-Satorras, R., and Vespignani, A. (2001). Epidemic dynamics and endemic states in complex networks. Phys. Rev. E Stat. Nonlin. Soft Matter Phys. 63, 066117.

Paul, S. M., Mytelka, D. S., Dunwiddie, C. T., Persinger, C. C., Munos, B. H., Lindborg, S. R., and Schacht, A. L. (2010). How to improve R\&D productivity: the pharmaceutical industry's grand challenge. Nat. Rev. Drug Discov. 9, 203-214.

Podani, J., Oltvai, Z. N., Jeong, H., Tombor, B., Barabasi, A. L., and Szathmary, E. (2001). Comparable system-level organization of Archaea and Eukaryotes. Nat. Genet. 29, 54-56.

Rash, L. D., and Hodgson, W. C. (2002). Pharmacology and biochemistry of spider venoms. Toxicon 40, 225-254.

Rask-Andersen, M., Almen, M. S., and Schioth, H. B. (2011). Trends in the exploitation of novel drug targets. Nat. Rev. Drug Discov. 10, 579-590.

Rasmussen, K. (2006). Creating more effective antidepressants: clues from the clinic. Drug Discov. Today 11, 623-631.

Ravasz, E., Somera, A. L., Mongru, D. A., Oltvai, Z. N., and Barabasi, A. L. (2002). Hierarchical organization of modularity in metabolic networks. Science 297, 1551-1555.

Reijneveld, J. C., Ponten, S. C., Berendse, H. W., and Stam, C. J. (2007). The application of graph theoretical analysis to complex networks in the brain. Clin. Neurophysiol. 118, 2317-2331.

Reyes, S. A., Salvi, R. J., Burkard, R. F., Coad, M. L., Wack, D. S. Galantowicz, P. J., and Lockwood, A. H. (2002). Brain imaging of the effects of lidocaine on tinnitus. Hear. Res. 171, 43-50.

Rodriguez de la Vega, R. C., Schwartz, E. F., and Possani, L. D. (2010). Mining on scorpion venom biodiversity. Toxicon $56,1155-1161$.

Roth, B. L., Sheffler, D. J., and Kroeze, W. K. (2004). Magic shotguns versus magic bullets: selectively nonselective drugs for mood disorders and schizophrenia. Nat. Rev. Drug Discov. 3, 353-359.

Rubinov, M., and Bassett, D. S. (2011). Emerging evidence of connectomic abnormalities in schizophrenia. $J$. Neurosci. 31, 6263-6265.

Salvador, R., Suckling, J., Coleman, M. R., Pickard, J. D., Menon, D., and Bullmore, E. (2005). Neurophysiological architecture of functional magnetic resonance images of human brain. Cereb. Cortex 15, 1332-1342.

Sams-Dodd, F. (2005). Target-based drug discovery: is something wrong? Drug Discov. Today 10, 139-147.

Schlee, W., Hartmann, T., Langguth, B., and Weisz, N. (2009a). Abnormal resting-state cortical coupling in chronic tinnitus. BMC Neurosci. 10, 11.

Schlee, W., Mueller, N., Hartmann, T., Keil, J., Lorenz, I., and Weisz, N. (2009b). Mapping cortical hubs in tinnitus. BMC Biol. 7, 80.

Schlee, W., Weisz, N., Bertrand, O., Hartmann, T., and Elbert, T. (2008). Using auditory steady state responses to outline the functional connectivity in the tinnitus brain. PLoS One 3, e3720. doi: 10.1371/journal.pone.0003720 
Shargel, B., Sayama, H., Epstein, I. R., and Bar-Yam, Y. (2003). Optimization of robustness and connectivity in complex networks. Phys. Rev. Lett. 90, 068701.

Siemens, J., Zhou, S., Piskorowski, R., Nikai, T., Lumpkin, E. A., Basbaum, A. I., King, D., and Julius, D. (2006). Spider toxins activate the capsaicin receptor to produce inflammatory pain. Nature 444, 208-212.

Sporns, O. (2011a). The human connectome: a complex network. Ann. N.Y. Acad. Sci. 1224, 109-125.

Sporns, O. (ed). (2011b). Networks of the Brain. Cambridge, MA: MIT Press.

Sporns, O. (2011c). The non-random brain: efficiency, economy, and complex dynamics. Front. Comput. Neurosci. 5, 5. doi: 10.3389/fncom. 2011.00005

Stam, C., and van Dijk, B. (2002). Synchronization likelihood: an unbiased measure of generalized synchronization in multivariate data sets. Physica. D 163, 236-251.

Stam, C. J. (2004). Functional connectivity patterns of human magnetoencephalographic recordings: a 'small-world' network? Neurosci. Lett 355, 25-28.

Suckfull, M., Althaus, M., Ellers-Lenz, B., Gebauer, A., Gortelmeyer, R., Jastreboff, P. J., Moebius, H. J., Rosenberg, T., Russ, H., Wirth, Y., and Krueger, H. (2011). A randomized, double-blind, placebocontrolled clinical trial to evaluate the efficacy and safety of neramexane in patients with moderate to severe subjective tinnitus. BMC Ear Nose Throat Disord. 11, 1.

Sullivan, M., Katon, W., Russo, J., Dobie, R., and Sakai, C. (1993). A randomized trial of nortriptyline for severe chronic tinnitus. Effects on depression, disability, and tinnitus symptoms. Arch. Intern. Med. 153, 2251-2259.

Szabadics, J., Varga, C., Molnar, G., Olah, S., Barzo, P., and Tamas, G. (2006). Excitatory effect of GABAergic axo-axonic cells in cortical microcircuits. Science 311, 233-235.

Tong, A. H., Lesage, G., Bader, G. D., Ding, H., Xu, H., Xin, X., Young, J., Berriz, G. F., Brost, R. L., Chang, M., Chen, Y., Cheng, X., Chua, G., Friesen, H., Goldberg, D. S., Haynes, J., Humphries, C., He, G., Hussein, S., Ke, L., Krogan, N., Li, Z., Levinson, J. N., Lu, H., Menard, P., Munyana, C., Parsons, A. B., Ryan, O., Tonikian, R., Roberts, T., Sdicu, A. M., Shapiro, J., Sheikh, B., Suter, B., Wong, S. L., Zhang, L. V., Zhu, H., Burd, C. G., Munro, S., Sander, C., Rine, J., Greenblatt, J., Peter, M., Bretscher, A., Bell, G., Roth, F. P., Brown, G. W., Andrews, B., Bussey, H., and Boone, C. (2004). Global mapping of the yeast genetic interaction network. Science 303, 808-813.

Trellakis, S., Lautermann, J., and Lehnerdt, G. (2007). Lidocaine: neurobiological targets and effects on the auditory system. Prog. Brain Res. 166, 303-322.

Uhlhaas, P. J., and Singer, W. (2006). Neural synchrony in brain disorders: relevance for cognitive dysfunctions and pathophysiology. Neuron 52, 155-168.

Uma, B., Swaroopa Rani, T., and Podile, A. R. (2011). Warriors at the gate that never sleep: non-host resistance in plants. J. Plant Physiol. 168, 2141-2152.

Valente, A. X., Sarkar, A., and Stone, H. A. (2004). Two-peak and three-peak optimal complex networks. Phys. Rev. Lett. 92, 118702.

van de Ville, D., Britz, J., and Michel, C. M. (2010). EEG microstate sequences in healthy humans at rest reveal scale-free dynamics. Proc. Natl. Acad. Sci. U.S.A. 107, 18179-18184.

van Dellen, E., Douw, L., Baayen, J. C., Heimans, J. J., Ponten, S. C., Vandertop, W. P., Velis, D. N., Stam, C. J., and Reijneveld, J. C. (2009). Long-term effects of temporal lobe epilepsy on local neural networks: a graph theoretical analysis of corticography recordings. PLoS
One 4, e8081. doi: 10.1371/journal.pone. 0008081

van den Heuvel, M. P., Mandl, R. C., Stam, C. J., Kahn, R. S., and Hulshoff Pol, H. E. (2010). Aberrant frontal and temporal complex network structure in schizophrenia: a graph theoretical analysis. $J$. Neurosci. 30, 15915-15926.

van den Heuvel, M. P., and Sporns, O. (2011). Rich-club organization of the human connectome. J. Neurosci. 31, 15775-15786.

van den Heuvel, M. P., Stam, C. J., Boersma, M., and Hulshoff Pol, H. E. (2008). Small-world and scalefree organization of voxel-based resting-state functional connectivity in the human brain. Neuroimage 43 , 528-539.

van der Loo, E., Gais, S., Congedo, M., Vanneste, S., Plazier, M. Menovsky, T., Van De Heyning, P., and De Ridder, D. (2009). Tinnitus intensity dependent gamma oscillations of the contralateral auditory cortex. PLoS One 4, e7396. doi: 10.1371/journal.pone. 0007396

Vanneste, S., Plazier, M., Der Loo, E., De Heyning, P. V., Congedo, M., and De Ridder, D. (2010). The neural correlates of tinnitusrelated distress. Neuroimage 52, 470-480.

Vio, M. M., and Holme, R. H. (2005). Hearing loss and tinnitus: 250 million people and a US\$10 billion potential market. Drug Discov. Today 10, 1263-1265.

Wang, L., Zhu, C., He, Y., Zang, Y., Cao, Q., Zhang, H., Zhong, Q., and Wang, Y. (2009). Altered smallworld brain functional networks in children with attention-deficit/ hyperactivity disorder. Hum. Brain Mapp. 30, 638-649.

Watts, D. J. (2002). A simple model of global cascades on random networks. Proc. Natl. Acad. Sci. U.S.A. 99, 5766-5771.

Watts, D. J., and Strogatz, S. H. (1998). Collective dynamics of 'small-world' networks. Nature 393 440-442.
Weinstock, M., Luques, L., Bejar, C., and Shoham, S. (2006). Ladostigil, a novel multifunctional drug for the treatment of dementia co-morbid with depression. J. Neural Transm. Suppl. 70, 443-446.

Weisz, N., Moratti, S., Meinzer, M., Dohrmann, K., and Elbert, T. (2005). Tinnitus perception and distress is related to abnormal spontaneous brain activity as measured by magnetoencephalography. PLoS Med. 2, e153. doi: 10.1371/journal.pmed.0020153

Weisz, N., Muller, S., Schlee, W., Dohrmann, K., Hartmann, T., and Elbert, T. (2007). The neural code of auditory phantom perception. $J$. Neurosci. 27, 1479-1484.

Wuchty, S., Oltvai, Z. N., and Barabasi, A. L. (2003). Evolutionary conservation of motif constituents in the yeast protein interaction network. Nat. Genet. 35, 176-179.

Yildirim, M. A., Goh, K. I., Cusick, M. E., Barabasi, A. L., and Vidal, M. (2007). Drug-target network. Nat. Biotechnol. 25, 1119-1126.

Conflict of Interest Statement: The authors declare that the research was conducted in the absence of any commercial or financial relationships that could be construed as a potential conflict of interest.

Received: 08 December 2011; accepted: 11 January 2012; published online: 25 January 2012.

Citation: Elgoyhen $A B$, Langguth $B$, Vanneste S and De Ridder D (2012) Tinnitus: network pathophysiologynetwork pharmacology. Front. Syst. Neurosci. 6:1. doi: 10.3389/fnsys. 2012.00001

Copyright () 2012 Elgoyhen, Langguth, Vanneste and De Ridder. This is an openaccess article distributed under the terms of the Creative Commons Attribution Non Commercial License, which permits non-commercial use, distribution, and reproduction in other forums, provided the original authors and source are credited. 